Research Article

\title{
Permanence and Global Attractivity of a Delayed Discrete Predator-Prey System with General Holling-Type Functional Response and Feedback Controls
}

\author{
Lijuan Chen, Junyan Xu, and Zhong Li \\ College of Mathematics and Computer Science, Fuzhou University, Fuzhou, Fujian 350002, China \\ Correspondence should be addressed to Lijuan Chen, chenlijuan@fzu.edu.cn
}

Received 8 June 2008; Accepted 11 September 2008

Recommended by Leonid Berezansky

This paper discusses a delayed discrete predator-prey system with general Holling-type functional response and feedback controls. Firstly, sufficient conditions are obtained for the permanence of the system. After that, under some additional conditions, we show that the periodic solution of the system is global stable.

Copyright (C) 2008 Lijuan Chen et al. This is an open access article distributed under the Creative Commons Attribution License, which permits unrestricted use, distribution, and reproduction in any medium, provided the original work is properly cited.

\section{Introduction}

The following predator-prey system with Holling-type II functional response and delays

$$
\begin{aligned}
& \dot{x}_{1}(t)=x_{1}(t)\left[r_{1}(t)-a_{11}(t) x_{1}\left(t-\tau_{1}(t)\right)-\frac{a_{12}(t) x_{2}(t)}{1+m x_{1}(t)}\right] \\
& \dot{x}_{2}(t)=x_{2}(t)\left[-r_{2}(t)-\frac{a_{21}(t) x_{1}\left(t-\tau_{2}(t)\right)}{1+m x_{1}\left(t-\tau_{2}(t)\right)}-a_{22}(t) x_{2}\left(t-\tau_{3}(t)\right)\right],
\end{aligned}
$$

and some generalized systems of general Holling-type functional response have been studied by many scholars (see [1-3] and the references cited therein). It has been found that the discrete time models governed by difference equations are more appropriate than the continuous ones when the populations have non-overlapping generations. Discrete time models can also provide efficient computational models of continuous models for numerical 
simulations (see [4-12]). In [4], Yang considered the following delayed discrete predator-prey system with general Holling-type functional response:

$$
\begin{aligned}
& N_{1}(k+1)=N_{1}(k) \exp \left\{r(k)-b(k) N_{1}^{\theta}\left(k-\left[\tau_{1}(t)\right]\right)-\frac{\alpha(k) N_{1}^{p-1}(k)}{1+m N_{1}^{p}(k)} N_{2}(k-[\sigma(t)])\right\}, \\
& N_{2}(k+1)=N_{2}(k) \exp \left\{-d(k)-a(k) N_{2}\left(k-\left[\tau_{2}(t)\right]\right)+\frac{\beta(k) N_{1}^{p}\left(k-\left[\tau_{3}(t)\right]\right)}{1+m N_{1}^{p}\left(k-\left[\tau_{3}(t)\right]\right)}\right\} .
\end{aligned}
$$

Sufficient conditions which guarantee the existence of at least one positive periodic solution are obtained by using the continuation theorem of coincidence degree theory. But Yang did not consider the permanence and globally attractivity of system (1.2), which are two of the most important topics in the study of population dynamics.

On the other hand, as was pointed out by Huo and Li [13], ecosystem in the real world is continuously distributed by unpredictable forces which can result in changes in the biological parameters such as survival rates. Of practical interest in ecology is the question of whether or not an ecosystem can withstand those unpredictable disturbances which persist for a finite period of time. In the language of control variables, we call the disturbance functions as control variables (for more discussion on this section, one could refer to [1216] for more details). Though much works dealt with the continuous time model. However, to the best of the author's knowledge, up to this day, there are still no scholars that propose and study the system (1.2) with feedback control. Therefore, the main purpose of this paper is to study the following delayed discrete predator-prey system with general Holling-type functional response and feedback control:

$$
\begin{aligned}
x_{1}(k+1) & =x_{1}(k) \exp \left\{r_{1}(k)-b_{1}(k) x_{1}^{\theta}\left(k-\tau_{1}\right)-\frac{\alpha_{1}(k) x_{1}^{p-1}(k)}{1+m x_{1}^{p}(k)} x_{2}\left(k-\tau_{3}\right)-e_{1}(k) u_{1}(k)\right\}, \\
x_{2}(k+1) & =x_{2}(k) \exp \left\{-r_{2}(k)-b_{2}(k) x_{2}\left(k-\tau_{2}\right)+\frac{\alpha_{2}(k) x_{1}^{p}\left(k-\tau_{4}\right)}{1+m x_{1}^{p}\left(k-\tau_{4}\right)}-e_{2}(k) u_{2}(k)\right\}, \\
\Delta u_{1}(k) & =-\eta_{1}(k) u_{1}(k)+q_{1}(k) x_{1}(k), \\
\Delta u_{2}(k) & =-\eta_{2}(k) u_{2}(k)+q_{2}(k) x_{2}(k),
\end{aligned}
$$

where $x_{1}(k)$ is the density of prey species at $k$ th generation, $x_{2}(k)$ is the density of predator species at $k$ th generation, $u_{1}(k)$ and $u_{2}(k)$ are control variables. Also, $r_{1}(k), b_{1}(k)$ denote the intrinsic growth rate and density-dependent coefficient of the prey, respectively, $r_{2}(k), b_{2}(k)$ denote the death rate and density-dependent coefficient of the predator, $\alpha_{1}(k)$ denote the capturing rate of the predator, $\alpha_{2}(k) / \alpha_{1}(k)$ represent the rate of conversion of nutrients into the reproduction of the predator. Further, $\tau_{i}(i=1,2,3,4)$ are nonnegative constants and $m, p$ are positive constants. In this paper, we always assume that $\left\{r_{i}(k)\right\},\left\{b_{i}(k)\right\},\left\{\alpha_{1}(k)\right\},\left\{e_{i}(k)\right\},\left\{\eta_{i}(k)\right\},\left\{q_{i}(k)\right\}, i=1,2$, are bounded nonnegative sequences and

$$
0<\eta_{i}^{L} \leq \eta_{i}^{M}<1, \quad i=1,2
$$


Here, for any bounded sequence $\{a(k)\}, a^{M}=\sup _{k \in N}\{a(k)\}$, and $a^{L}=\inf _{k \in N}\{a(k)\}$, where $N=\{0,1,2, \ldots\}$.

This paper is organized as follows. In Section 2, we will introduce some definition and establish several useful lemma. The permanence of system (1.3) is then studied in Section 3. In Section 4, based on the permanence result, under the assumption that all the delays are equal to zero and the coefficients of the system are periodic sequences, we obtain a set of sufficient conditions which guarantee the existence and stability of a unique globally attractive positive periodic solution of the system.

By the biological meaning, we will focus our discussion on the positive solution of system (1.3). So it is assumed that the initial conditions of (1.3) are of the form

$$
x_{i}(-k) \geq 0, \quad u_{i}(-k) \geq 0, \quad k \in N \cap(0, \tau], \quad x_{i}(0)>0, \quad u_{i}(0)>0, \quad i=1,2,
$$

where $\tau=\max \left\{\tau_{1}, \tau_{2}, \tau_{3}, \tau_{4}\right\}$.

One can easily show that the solutions of (1.3) with the initial condition (1.5) are defined and remain positive for all $k \in N$.

\section{Preliminaries}

In this section, we will introduce the definition of permanence and several useful lemmas.

Definition 2.1. System (1.3) is said to be permanent if there exist positive constants $x_{i}^{*}, u_{i}^{*}, x_{i *}, u_{i *}$, which are independent of the solution of the system, such that for any positive solution $\left(x_{1}(k), x_{2}(k), u_{1}(k), u_{2}(k)\right)$ of system (1.3) satisfies

$$
\begin{aligned}
& x_{i *} \leq \liminf _{k \rightarrow \infty} x_{i}(k) \leq \limsup _{k \rightarrow \infty} x_{i}(k) \leq x_{i}^{*}, \\
& u_{i *} \leq \liminf _{k \rightarrow \infty} u_{i}(k) \leq \limsup _{k \rightarrow \infty} u_{i}(k) \leq u_{i}^{*},
\end{aligned}
$$

for $i=1,2$.

Lemma 2.2. Assume that $x(k)$ satisfies

$$
x(k+1) \leq x(k) \exp \left\{a(k)-b(k) x^{\theta}(k)\right\} \quad \forall k \geq k_{0},
$$

where $\{a(k)\}$ and $\{b(k)\}$ are positive sequences, $x\left(k_{0}\right)>0, \theta$ is a positive constant, and $k_{0} \in N$. Then one has

$$
\limsup _{k \rightarrow \infty} x(k) \leq D
$$

where $D=\left(1 / \theta b^{L}\right)^{1 / \theta} \exp \left(a^{M}-1 / \theta\right)$.

Lemma 2.3. Assume that $x(k)$ satisfies

$$
x(k+1) \geq x(k) \exp \left\{a(k)-b(k) x^{\theta}(k)\right\} \quad \forall k \geq k_{0},
$$


where $\{a(k)\}$ and $\{b(k)\}$ are positive sequences, $x\left(k_{0}\right)>0, \theta$ is a positive constant, and $k_{0} \in N$. Also, $\lim \sup _{k \rightarrow \infty} x(k) \leq D$ and $b^{M} D^{\theta} / a^{L}>1$. Then one has

$$
\liminf _{k \rightarrow \infty} x(k) \geq C,
$$

where $C=\left(a^{L} / b^{M}\right)^{1 / \theta} \exp \left(a^{L}-b^{M} D^{\theta}\right)$.

Proof. The proofs of Lemmas 2.2 and 2.3 are very similar to those of [6, Propositions 2.1 and 2.2], respectively. So we omit the detail here.

Lemma 2.4. Assume that $x(k)$ satisfies

$$
x(k+1) \leq x(k) \exp \left\{a(k)-b(k) x^{\theta}(k-\tau)\right\} \quad \forall k \geq k_{0}>\tau,
$$

where $\{a(k)\}$ and $\{b(k)\}$ are positive sequences, $x\left(k_{0}\right)>0, \theta$ and $\tau$ are positive constants, and $k_{0} \in N$. Then one has

$$
\limsup _{k \rightarrow \infty} x(k) \leq B
$$

where $B=\left(1 / \theta \beta^{L}\right)^{1 / \theta} \exp \left(a^{M}-1 / \theta\right)$ and $\beta(k)=b(k) \exp \left\{-\theta \sum_{i=k-\tau}^{k-1} a(i)\right\}$.

Proof. From the above equation, one has

$$
x(k+1) \leq x(k) \exp \{a(k)\} \quad \forall k \geq k_{0}
$$

Sequently we can easily obtain that

$$
x(k-\tau) \geq x(k) \exp \left\{-\sum_{i=k-\tau}^{k-1} a(i)\right\} .
$$

So one has

$$
\begin{aligned}
x(k+1) & \leq x(k) \exp \left\{a(k)-b(k) \exp \left\{-\theta \sum_{i=k-\tau}^{k-1} a(i)\right\} x^{\theta}(k)\right\} \\
& =x(k) \exp \left\{a(k)-\beta(k) x^{\theta}(k)\right\} .
\end{aligned}
$$

By Lemma 2.2, we can complete the proof of Lemma 2.4.

Lemma 2.5. Assume that $x(k)$ satisfies

$$
x(k+1) \geq x(k) \exp \left\{a(k)-b(k) x^{\theta}(k-\tau)\right\} \quad \forall k \geq k_{0}>\tau,
$$


where $\{a(k)\}$ and $\{b(k)\}$ are positive sequences, $x\left(k_{0}\right)>0, \theta$ and $\tau$ are positive constants, and $k_{0} \in N$. Also, $\lim \sup _{k \rightarrow \infty} x(k) \leq B$ and $\gamma^{M} B^{\theta} / a^{L}>1$, where $\gamma(k)=b(k) \exp \left\{-\theta \sum_{i=k-\tau}^{k-1}(a(i)-\right.$ $\left.\left.b(i) B^{\theta}\right)\right\}$. Then one has

$$
\liminf _{k \rightarrow \infty} x(k) \geq A,
$$

where $A=\left(a^{L} / \gamma^{M}\right)^{1 / \theta} \exp \left(a^{L}-\gamma^{M} B^{\theta}\right)$.

Proof. From the above equation, one has

$$
x(k+1) \geq x(k) \exp \left\{a(k)-b(k) D^{\theta}\right\} \quad \forall k \geq k_{0} .
$$

Sequently we can easily obtain that

$$
x(k-\tau) \leq x(k) \exp \left\{-\sum_{i=k-\tau}^{k-1}\left(a(i)-b(i) D^{\theta}\right)\right\} .
$$

So one has

$$
\begin{aligned}
x(k+1) & \geq x(k) \exp \left\{a(k)-b(k) \exp \left\{-\theta \sum_{i=k-\tau}^{k-1}\left(a(i)-b(i) D^{\theta}\right)\right\} x^{\theta}(k)\right\} \\
& =x(k) \exp \left\{a(k)-\gamma(k) x^{\theta}(k)\right\} .
\end{aligned}
$$

By Lemma 2.3, we can complete the proof of Lemma 2.5 . Wang.

Lemma 2.6 is a direct corollary of [17, Theorem 6.2, page 125] by L. Wang and M. Q.

Lemma 2.6. Consider the following first-order difference equation:

$$
y(k+1)=A y(k)+B, \quad k=1,2 \ldots,
$$

where $A, B$ are positive constants. Assuming $A<1$, for any solution $\{y(k)\}$ of the above system, one has

$$
\lim _{k \rightarrow \infty} y(k)=\frac{B}{1-A}
$$

The following comparison theorem for the difference equation is of [17, Theorem 2.1, page 241] by L. Wang and M. Q. Wang. 
Lemma 2.7. Let $k \in\left\{k_{0}, k_{0}+1, \ldots, k_{0}+l, \ldots\right\}, r \geq 0$. For fixed $k, g(k, r)$ is a nondecreasing function with respect to $r$, and for $k \geq k_{0}$, the following inequalities hold:

$$
\begin{aligned}
& y(k+1) \leq g(k, y(k)), \\
& u(k+1) \geq g(k, u(k)) .
\end{aligned}
$$

If $y\left(k_{0}\right) \leq u\left(k_{0}\right)$, then $y(k) \leq u(k)$ for all $k \geq k_{0}$.

\section{Permanence}

In this section, we establish a permanent result for system (1.3).

Proposition 3.1. In addition to (1.4), assume further that

$\left(H_{1}\right)$

$$
\left(\frac{\alpha_{2}(k)}{m}-r_{2}(k)\right)^{L}>0
$$

for any positive solution $\left(x_{1}(k), x_{2}(k), u_{1}(k), u_{2}(k)\right)$ of system $(1.3)$, one has

$$
\limsup _{k \rightarrow \infty} x_{i}(k) \leq x_{i}^{*}, \quad \limsup _{k \rightarrow \infty} u_{i}(k) \leq u_{i}^{*}, \quad i=1,2,
$$

where

$$
\begin{gathered}
x_{1}^{*}=\left(\frac{1}{\theta \beta_{1}^{L}}\right)^{1 / \theta} \exp \left(r_{1}^{M}-\frac{1}{\theta}\right), \quad \beta_{1}(k)=b_{1}(k) \exp \left\{-\theta \sum_{i=k-\tau_{1}}^{k-1} r_{1}(i)\right\}, \\
x_{2}^{*}=\frac{1}{\beta_{2}^{L}} \exp \left(\left(\frac{\alpha_{2}(k)}{m}-r_{2}(k)\right)^{M}-1\right), \quad \beta_{2}(k)=b_{2}(k) \exp \left\{-\sum_{i=k-\tau_{2}}^{k-1}\left(\frac{\alpha_{2}(i)}{m}-r_{2}(i)\right)\right\}, \\
u_{1}^{*}=\frac{q_{1}^{M} x_{1}^{*}}{\eta_{1}^{L}}, \quad u_{2}^{*}=\frac{q_{2}^{M} x_{2}^{*}}{\eta_{2}^{L}} .
\end{gathered}
$$

Proof. Let $\left(x_{1}(k), x_{2}(k), u_{1}(k), u_{2}(k)\right)$ be any positive solution of system (1.3), from the first equation of (1.3), it follows that

$$
x_{1}(k+1) \leq x_{1}(k) \exp \left\{r_{1}(k)-b_{1}(k) x_{1}^{\theta}\left(k-\tau_{1}\right)\right\}
$$

By applying Lemmas 2.4 and 2.7, we obtain

$$
\limsup _{k \rightarrow \infty} x_{1}(k) \leq x_{1}^{*}
$$


where

$$
x_{1}^{*}=\left(\frac{1}{\theta \beta_{1}^{L}}\right)^{1 / \theta} \exp \left(r_{1}^{M}-\frac{1}{\theta}\right), \quad \beta_{1}(k)=b_{1}(k) \exp \left\{-\theta \sum_{i=k-\tau_{1}}^{k-1} r_{1}(i)\right\}
$$

Similarly, from the second equation of (1.3), it follows that

$$
x_{2}(k+1) \leq x_{2}(k) \exp \left\{\frac{\alpha_{2}(k)}{m}-r_{2}(k)-b_{2}(k) x_{2}\left(k-\tau_{2}\right)\right\} .
$$

Under the assumption $\left(H_{1}\right)$, by applying Lemmas 2.4 and 2.7 , we obtain

$$
\limsup _{k \rightarrow \infty} x_{2}(k) \leq x_{2}^{*}
$$

where

$$
x_{2}^{*}=\frac{1}{\beta_{2}^{L}} \exp \left(\left(\frac{\alpha_{2}(k)}{m}-r_{2}(k)\right)^{M}-1\right), \quad \beta_{2}(k)=b_{2}(k) \exp \left\{-\sum_{i=k-\tau_{2}}^{k-1}\left(\frac{\alpha_{2}(i)}{m}-r_{2}(i)\right)\right\} .
$$

For any positive constant $\varepsilon$ small enough, it follows from (3.5) and (3.8) that there exists large enough $K_{1}>\tau$ such that

$$
x_{1}(k) \leq x_{1}^{*}+\varepsilon, \quad x_{2}(k) \leq x_{2}^{*}+\varepsilon \quad \forall k \geq K_{1} .
$$

Then the third equation of (1.3) leads to

$$
\Delta u_{1}(k) \leq-\eta_{1}(k) u_{1}(k)+q_{1}(k)\left(x_{1}^{*}+\varepsilon\right) .
$$

And so

$$
u_{1}(k+1) \leq\left(1-\eta_{1}^{L}\right) u_{1}(k)+q_{1}^{M}\left(x_{1}^{*}+\varepsilon\right) \quad \forall k \geq K_{1} .
$$

By applying Lemmas 2.6 and 2.7, it follows from (3.12) that

$$
\limsup _{k \rightarrow \infty} u_{1}(k) \leq \frac{q_{1}^{M}\left(x_{1}^{*}+\varepsilon\right)}{\eta_{1}^{L}} .
$$

Setting $\varepsilon \rightarrow 0$ in the above inequality leads to

$$
\limsup _{k \rightarrow \infty} u_{1}(k) \leq u_{1}^{*}
$$


where $u_{1}^{*}=q_{1}^{M} x_{1}^{*} / \eta_{1}^{L}$. Similarly, we can obtain

$$
\limsup _{k \rightarrow \infty} u_{2}(k) \leq u_{2}^{*}
$$

where $u_{2}^{*}=q_{2}^{M} x_{2}^{*} / \eta_{2}^{L}$. Thus we complete the proof of Proposition 3.1.

Proposition 3.2. In addition to (1.4), assume further that

$\left(H_{2}\right)$

$$
r_{1}^{L}-\alpha_{1}^{M}\left(x_{1}^{*}\right)^{p-1} x_{2}^{*}-e_{1}^{M} u_{1}^{*}>0,
$$

$\left(H_{3}\right)$

$$
-r_{2}^{M}+\frac{\alpha_{2}^{L} x_{1 *}^{p}}{1+m x_{1 *}^{p}}-e_{2}^{M} u_{2}^{*}>0
$$

for any positive solution $\left(x_{1}(k), x_{2}(k), u_{1}(k), u_{2}(k)\right)$ of system (1.3), there exist positive constants $x_{i *}, u_{i *}$, such that

$$
\liminf _{k \rightarrow \infty} x_{i}(k) \geq x_{i *}, \quad \liminf _{k \rightarrow \infty} u_{i}(k) \geq u_{i *,} \quad i=1,2
$$

Proof. Let $\left(x_{1}(k), x_{2}(k), u_{1}(k), u_{2}(k)\right)$ be any positive solution of system (1.3). From $\left(H_{2}\right)$ and $\left(H_{3}\right)$, there exists a small enough positive constant $\varepsilon$ such that

$$
\begin{gathered}
r_{1}^{L}-\alpha_{1}^{M}\left(x_{1}^{*}+\varepsilon\right)^{p-1}\left(x_{2}^{*}+\varepsilon\right)-e_{1}^{M}\left(u_{1}^{*}+\varepsilon\right)>0 \\
-r_{2}^{M}+\frac{\alpha_{2}^{L}\left(x_{1 *}-\varepsilon\right)^{p}}{1+m\left(x_{1 *}-\varepsilon\right)^{p}}-e_{2}^{M}\left(u_{2}^{*}+\varepsilon\right)>0 .
\end{gathered}
$$

Also, according to Proposition 3.1, for the above $\varepsilon$, there exists $K_{2}>K_{1}$ such that for $k \geq K_{2}$,

$$
\begin{array}{ll}
x_{1}(k) \leq x_{1}^{*}+\varepsilon, & x_{2}(k) \leq x_{2}^{*}+\varepsilon \\
u_{1}(k) \leq u_{1}^{*}+\varepsilon, & u_{2}(k) \leq u_{2}^{*}+\varepsilon
\end{array}
$$

Then from the first equation of (1.3), one has

$$
\begin{aligned}
x_{1}(k+1) & \geq x_{1}(k) \exp \left\{r_{1}(k)-b_{1}(k) x_{1}^{\theta}\left(k-\tau_{1}\right)-\alpha_{1}(k) x_{1}^{p-1}(k) x_{2}\left(k-\tau_{3}\right)-e_{1}(k) u_{1}(k)\right\} \\
& \geq x_{1}(k) \exp \left\{r_{1}(k)-\alpha_{1}(k)\left(x_{1}^{*}+\varepsilon\right)^{p-1}\left(x_{2}^{*}+\varepsilon\right)-e_{1}(k)\left(u_{1}^{*}+\varepsilon\right)-b_{1}(k) x_{1}^{\theta}\left(k-\tau_{1}\right)\right\}
\end{aligned}
$$


Let $a_{1}(k, \varepsilon)=r_{1}(k)-\alpha_{1}(k)\left(x_{1}^{*}+\varepsilon\right)^{p-1}\left(x_{2}^{*}+\varepsilon\right)-e_{1}(k)\left(u_{1}^{*}+\varepsilon\right)$, so the above inequality follows that

$$
x_{1}(k+1) \geq x_{1}(k) \exp \left\{a_{1}(k, \varepsilon)-b_{1}(k) x_{1}^{\theta}\left(k-\tau_{1}\right)\right\}
$$

Consequently, let $\gamma_{1}(k, \varepsilon)=b_{1}(k) \exp \left\{-\theta \sum_{i=k-\tau_{1}}^{k-1}\left(a_{1}(i, \varepsilon)-b_{1}(i) x_{1}^{* \theta}\right)\right\}$. Because $\gamma_{1}^{M}>\beta_{1}^{L}$, one has

$$
\frac{r_{1}^{M}}{a_{1}^{L}}\left(x_{1}^{*}\right)^{\theta}=\frac{r_{1}^{M}}{a_{1}^{L}} \frac{\exp \left(\theta r_{1}^{M}-1\right)}{\theta \beta_{1}^{L}}>1
$$

Here we use the fact that $\exp \left(\theta r_{1}^{M}-1\right)>\theta r_{1}^{M}>\theta a_{1}^{M}>\theta a_{1}^{L}$. From (3.19) and (3.23), by Lemmas 2.5 and 2.7, one has

$$
\liminf _{k \rightarrow \infty} x_{1}(k) \geq\left(\frac{a_{1}^{L}(\varepsilon)}{\gamma_{1}^{M}(\varepsilon)}\right)^{1 / \theta} \exp \left\{a_{1}^{L}(\varepsilon)-\gamma_{1}^{M}(\varepsilon)\left(x_{1}^{*}\right)^{\theta}\right\} .
$$

Setting $\varepsilon \rightarrow 0$ in the above inequality leads to

$$
\liminf _{k \rightarrow \infty} x_{1}(k) \geq x_{1 * \prime}
$$

where

$$
\begin{gathered}
x_{1 *}=\left(\frac{a_{1}^{L}}{r_{1}^{M}}\right)^{1 / \theta} \exp \left\{a_{1}^{L}-r_{1}^{M}\left(x_{1}^{*}\right)^{\theta}\right\}, \\
a_{1}(k)=r_{1}(k)-\alpha_{1}(k)\left(x_{1}^{*}\right)^{p-1} x_{2}^{*}-e_{1}(k) u_{1}^{*},
\end{gathered}
$$

and $\gamma_{1}(k)=b_{1}(k) \exp \left\{-\theta \sum_{i=k-\tau_{1}}^{k-1}\left(a_{1}(i)-b_{1}(i) x_{1}^{* \theta}\right)\right\}$.

Similarly, from the second equation of (1.3), one has

$$
x_{2}(k+1) \geq x_{2}(k) \exp \left\{-r_{2}(k)+\frac{\alpha_{2}(k)\left(x_{1 *}-\varepsilon\right)^{p}}{1+m\left(x_{1 *}-\varepsilon\right)^{p}}-e_{2}(k)\left(u_{2}^{*}+\varepsilon\right)-b_{2}(k) x_{2}\left(k-\tau_{2}\right)\right\} .
$$

Let $a_{2}(k, \varepsilon)=-r_{2}(k)+\alpha_{2}(k)\left(x_{1 *}-\varepsilon\right)^{p} /\left(1+m\left(x_{1 *}-\varepsilon\right)^{p}\right)-e_{2}(k)\left(u_{2}^{*}+\varepsilon\right)$, so the above inequality leads to

$$
x_{2}(k+1) \geq x_{2}(k) \exp \left\{a_{2}(k, \varepsilon)-b_{2}(k) x_{2}\left(k-\tau_{2}\right)\right\} .
$$

Consequently, let $\gamma_{2}(k, \varepsilon)=b_{2}(k) \exp \left\{-\sum_{i=k-\tau_{2}}^{k-1}\left(a_{2}(i, \varepsilon)-b_{2}(i) x_{2}^{*}\right)\right\}$. Because $\gamma_{2}^{M}>\beta_{2}^{L}$, one has

$$
\frac{r_{2}^{M}}{a_{2}^{L}} x_{2}^{*}=\frac{r_{2}^{M}}{a_{2}^{L}} \frac{\exp \left\{\left(\alpha_{2}(k) / m-r_{2}(k)\right)^{M}-1\right\}}{\beta_{2}^{L}}>1 .
$$


Here we use the fact that $\exp \left\{\left(\alpha_{2}(k) / m-r_{2}(k)\right)^{M}-1\right\}>\left(\alpha_{2}(k) / m-r_{2}(k)\right)^{M}>a_{2}^{M}>a_{2}^{L}$. From (3.20) and (3.29), by Lemmas 2.5 and 2.7, one has

$$
\liminf _{k \rightarrow \infty} x_{2}(k) \geq \frac{a_{2}^{L}(\varepsilon)}{r_{2}^{M}(\varepsilon)} \exp \left\{a_{2}^{L}(\varepsilon)-r_{2}^{M}(\varepsilon) x_{2}^{*}\right\} .
$$

Setting $\varepsilon \rightarrow 0$ in the above inequality leads to

$$
\liminf _{k \rightarrow \infty} x_{2}(k) \geq x_{2 *}
$$

where

$$
\begin{aligned}
x_{2 *} & =\frac{a_{2}^{L}}{r_{2}^{M}} \exp \left\{a_{2}^{L}-r_{2}^{M} x_{2}^{*}\right\}, \\
a_{2}(k) & =-r_{2}(k)+\frac{\alpha_{2}(k) x_{1 *}^{p}}{1+m x_{1 *}^{p}}-e_{2}(k) u_{2}^{*} \\
r_{2}(k) & =b_{2}(k) \exp \left\{-\sum_{i=k-\tau_{2}}^{k-1}\left(a_{2}(i)-b_{2}(i) x_{2}^{*}\right)\right\} .
\end{aligned}
$$

Then the third equation of (1.3) leads to

$$
\Delta u_{1}(k) \geq-\eta_{1}(k) u_{1}(k)+q_{1}(k)\left(x_{1 *}-\varepsilon\right) .
$$

And so

$$
u_{1}(k+1) \geq\left(1-\eta_{1}^{M}\right) u_{1}(k)+q_{1}^{L}\left(x_{1 *}-\varepsilon\right) \quad \forall k \geq K_{2} .
$$

By applying Lemmas 2.6 and 2.7, it follows from (3.35) that

$$
\limsup _{k \rightarrow \infty} u_{1}(k) \geq \frac{q_{1}^{L}\left(x_{1 *}-\varepsilon\right)}{\eta_{1}^{M}}
$$

Setting $\varepsilon \rightarrow 0$ in the above inequality leads to

$$
\limsup _{k \rightarrow \infty} u_{1}(k) \geq u_{1 *}
$$

where $u_{1 *}=q_{1}^{L} x_{1 *} / \eta_{1}^{M}$. Similarly, we can obtain

$$
\limsup _{k \rightarrow \infty} u_{2}(k) \leq u_{2 * \prime}
$$

where $u_{2 *}=q_{2}^{L} x_{2 *} / \eta_{2}^{M}$. Thus we complete the proof of Proposition 3.2. 
Theorem 3.3. In addition to (1.4), assume further that $\left(H_{1}\right),\left(H_{2}\right)$, and $\left(H_{3}\right)$ hold, then system (1.3) is permanent.

It should be noticed that, from the proofs of Propositions 3.1 and 3.2, we know that under the conditions of Theorem 3.3, the set $\Omega=\left\{\left(x_{1}, x_{2}, u_{1}, u_{2}\right) \mid x_{i *} \leq x_{i} \leq x_{i}^{*}, u_{i *} \leq u_{i} \leq\right.$ $\left.u_{i}^{*}, i=1,2\right\}$ is an invariant set of system (1.3).

\section{Existence and stability of a periodic solution}

In this section, we consider the stability property of system (1.3) under the assumption $\tau_{i}=$ $0(i=1,2,3,4)$, that is, we consider the following system:

$$
\begin{aligned}
x_{1}(k+1) & =x_{1}(k) \exp \left\{r_{1}(k)-b_{1}(k) x_{1}^{\theta}(k)-\frac{\alpha_{1}(k) x_{1}^{p-1}(k)}{1+m x_{1}^{p}(k)} x_{2}(k)-e_{1}(k) u_{1}(k)\right\}, \\
x_{2}(k+1) & =x_{2}(k) \exp \left\{-r_{2}(k)-b_{2}(k) x_{2}(k)+\frac{\alpha_{2}(k) x_{1}^{p}(k)}{1+m x_{1}^{p}(k)}-e_{2}(k) u_{2}(k)\right\}, \\
\Delta u_{1}(k) & =-\eta_{1}(k) u_{1}(k)+q_{1}(k) x_{1}(k), \\
\Delta u_{2}(k) & =-\eta_{2}(k) u_{2}(k)+q_{2}(k) x_{2}(k),
\end{aligned}
$$

which are similar to system (1.3) but do not include delays. In this section, we always assume that $\left\{r_{i}(k)\right\},\left\{b_{i}(k)\right\},\left\{\alpha_{1}(k)\right\},\left\{e_{i}(k)\right\},\left\{\eta_{i}(k)\right\},\left\{q_{i}(k)\right\}$ are bounded nonnegative periodic sequences with a common period $\omega$ and satisfy

$$
0<\eta_{i}(k)<1, \quad k \in N \cap[0, \omega], i=1,2 .
$$

Also it is assumed that the initial conditions of (4.1) are of the form

$$
x_{i}(0)>0, \quad u_{i}(0)>0, \quad i=1,2 .
$$

Using a similar way, under some conditions, we can obtain the permanence of system (4.1). As above, still let $x_{i}^{*}$ and $u_{i}^{*}, i=1,2$, be the upper bound of $\left\{x_{i}(k)\right\}$ and $\left\{u_{i}(k)\right\}, x_{i *}$ and let $u_{i *}, i=1,2$, be the lower bound of $\left\{x_{i}(k)\right\}$ and $\left\{u_{i}(k)\right\}$, where $x_{i}^{*}, u_{i}^{*}, x_{i *}$, and $u_{i *}$ are independent of the solution of system (4.1). Our first result concerns with the existence of a periodic solution.

Theorem 4.1. In addition to (4.2), assume further that $\left(H_{1}\right),\left(H_{2}\right)$, and $\left(H_{3}\right)$ hold, then system (4.1) has a periodic solution denoted by $\left\{\bar{x}_{1}(k), \bar{x}_{2}(k), \bar{u}_{1}(k), \bar{u}_{2}(k)\right\}$.

Proof. Let $\Omega=\left\{\left(x_{1}, x_{2}, u_{1}, u_{2}\right) \mid x_{i *} \leq x_{i} \leq x_{i}^{*}, u_{i *} \leq u_{i} \leq u_{i}^{*}, i=1,2\right\}, \Omega$ is an invariant set of system (4.1). Thus, we can define a mapping $F$ on $\Omega$ by

$$
F\left(x_{1}(0), x_{2}(0), u_{1}(0), u_{2}(0)\right)=\left(x_{1}(\omega), x_{2}(\omega), u_{1}(\omega), u_{2}(\omega)\right)
$$

for $\left(x_{1}(0), x_{2}(0), u_{1}(0), u_{2}(0)\right) \in \Omega$. 
Obviously, $F$ depends continuously on $\left(x_{1}(0), x_{2}(0), u_{1}(0), u_{2}(0)\right)$. Thus $F$ is continuous and maps a compact set $\Omega$ into itself. Therefore, $F$ has a fixed point $\left(\bar{x}_{1}, \bar{x}_{2}, \bar{u}_{1}, \bar{u}_{2}\right)$. It is easy to see that the solution $\left\{\bar{x}_{1}(k), \bar{x}_{2}(k), \bar{u}_{1}(k), \bar{u}_{2}(k)\right\}$ passing through $\left(\bar{x}_{1}, \bar{x}_{2}, \bar{u}_{1}, \bar{u}_{2}\right)$ is a periodic solution of system (4.1). This completes the proof.

Now, we study the globally stability property of the periodic solution obtained in Theorem 4.1.

Theorem 4.2. In addition to the conditions of Theorem 4.1, if system (4.1) satisfies

$$
\begin{gathered}
\lambda_{1}=\max \left\{\left|1-\theta b_{1}^{L} x_{1 *}^{\theta}\right|,\left|1-\theta b_{1}^{M}\left(x_{1}^{*}\right)^{\theta}-\alpha_{1}^{M} W_{1} x_{1}^{*}\right|\right\}+\alpha_{1}^{M} W_{2} x_{2}^{*}+e_{1}^{M}<1, \\
\lambda_{2}=\max \left\{\left|1-b_{2}^{L} x_{2 *}\right|,\left|1-b_{2}^{M} x_{2}^{*}\right|\right\}+\alpha_{2}^{M} W_{3} x_{1}^{*}+e_{2}^{M}<1, \\
\lambda_{3}=1-\eta_{1}^{L}+q_{1}^{M} x_{1}^{*}<1, \\
\lambda_{4}=1-\eta_{2}^{L}+q_{2}^{M} x_{2}^{*}<1,
\end{gathered}
$$

where the definition of $W_{i}, i=1,2,3$ can be seen in the following proof, then the w-periodic solution $\left(\bar{x}_{1}(k), \bar{x}_{2}(k), \bar{u}_{1}(k), \bar{u}_{2}(k)\right)$ obtained in Theorem 4.1 is globally attractive.

Proof. Assume that $\left(x_{1}(k), x_{2}(k), u_{1}(k), u_{2}(k)\right)$ is any positive solution of system $(4.1)$, let

$$
x_{i}(k)=\bar{x}_{i}(k) \exp \left\{y_{i}(k)\right\}, \quad u_{i}(k)=\bar{u}_{i}(k)+v_{i}(k), \quad i=1,2 .
$$

To complete the proof, it suffices to show that

$$
\lim _{k \rightarrow \infty} y_{i}(k)=0, \quad \lim _{k \rightarrow \infty} v_{i}(k)=0, \quad i=1,2
$$

Since

$$
\begin{aligned}
y_{1}(k+1)= & y_{1}(k)-b_{1}(k) \bar{x}_{1}^{\theta}(k)\left(\exp \left\{\theta y_{1}(k)\right\}-1\right)-e_{1}(k) v_{1}(k) \\
& -\alpha_{1}(k)\left[\frac{\bar{x}_{1}^{p-1}(k) \exp \left\{(p-1) y_{1}(k)\right\}}{1+m \bar{x}_{1}^{p}(k) \exp \left\{p y_{1}(k)\right\}} \bar{x}_{2}(k) \exp \left\{y_{2}(k)\right\}-\frac{\bar{x}_{1}^{p-1}(k)}{1+m \bar{x}_{1}^{p}(k)} \bar{x}_{2}(k)\right] \\
= & y_{1}(k)-b_{1}(k) \bar{x}_{1}^{\theta}(k) \exp \left\{\xi_{1}(k) \theta y_{1}(k)\right\} \theta y_{1}(k)-e_{1}(k) v_{1}(k) \\
& -\alpha_{1}(k)\left[f_{1}^{\prime}\left(\xi_{2}^{\prime}(k), x_{2}(k)\right) \bar{x}_{1}(k) \exp \left\{\xi_{4}(k) y_{1}(k)\right\} y_{1}(k)\right. \\
& \left.\quad+f_{2}^{\prime}\left(\bar{x}_{1}(k), \xi_{3}^{\prime}(k)\right) \bar{x}_{2}(k) \exp \left\{\xi_{5}(k) y_{2}(k)\right\} y_{2}(k)\right],
\end{aligned}
$$

where

$$
\begin{aligned}
f(x, y) & =\frac{x^{p-1} y}{1+m x^{p}}, \\
\xi_{2}^{\prime}(k) & =\bar{x}_{1}(k)+\xi_{2}(k)\left(x_{1}(k)-\bar{x}_{1}(k)\right), \\
\xi_{3}^{\prime}(k) & =\bar{x}_{2}(k)+\xi_{3}(k)\left(x_{2}(k)-\bar{x}_{2}(k)\right),
\end{aligned}
$$


and $\xi_{i}(k) \in(0,1)$ for $i=1,2,3,4,5$. Because of the boundedness of $\left\{\bar{x}_{1}(k)\right\},\left\{\bar{x}_{2}(k)\right\},\left\{x_{1}(k)\right\}$, $\left\{x_{2}(k)\right\},\left|f_{1}^{\prime}\left(\xi_{2}^{\prime}(k), x_{2}(k)\right)\right|,\left|f_{2}^{\prime}\left(\bar{x}_{1}(k), \xi_{3}^{\prime}(k)\right)\right|$ are bounded, where $f_{1}^{\prime}$ and $f_{2}^{\prime}$ mean the partial derivation of the function $f(x, y)$. Let $\left|f_{1}^{\prime}\left(\xi_{2}^{\prime}(k), x_{2}(k)\right)\right|<W_{1}$ and $\left|f_{2}^{\prime}\left(\bar{x}_{1}(k), \xi_{3}^{\prime}(k)\right)\right|<W_{2}$.

Similarly, we get

$$
\begin{aligned}
y_{2}(k+1)= & y_{2}(k)-b_{2}(k) \bar{x}_{2}(k)\left(\exp \left\{y_{2}(k)\right\}-1\right) \\
& +\alpha_{2}(k)\left[\frac{\bar{x}_{1}^{p}(k) \exp \left\{p y_{1}(k)\right\}}{1+m \bar{x}_{1}^{p}(k) \exp \left\{p y_{1}(k)\right\}}-\frac{\bar{x}_{1}^{p}(k)}{1+m \bar{x}_{1}^{p}(k)}\right]-e_{2}(k) v_{2}(k) \\
= & y_{2}(k)-b_{2}(k) \bar{x}_{2}(k) \exp \left\{\xi_{5}(k) y_{2}(k)\right\} y_{2}(k) \\
& +\alpha_{2}(k) g^{\prime}\left(\xi_{6}^{\prime}(k)\right) \bar{x}_{1}(k)\left(\exp \left\{y_{1}(k)\right\}-1\right)-e_{2}(k) v_{2}(k) \\
= & y_{2}(k)-b_{2}(k) \bar{x}_{2}(k) \exp \left\{\xi_{5}(k) y_{2}(k)\right\} y_{2}(k) \\
& +\alpha_{2}(k) g^{\prime}\left(\xi_{6}^{\prime}(k)\right) \bar{x}_{1}(k) \exp \left\{\xi_{4}(k) y_{1}(k)\right\} y_{1}(k)-e_{2}(k) v_{2}(k),
\end{aligned}
$$

where

$$
\xi_{6}^{\prime}(k)=\bar{x}_{1}(k)+\xi_{6}(k)\left(x_{1}(k)-\bar{x}_{1}(k)\right), \quad \xi_{6}(k) \in(0,1) .
$$

Because of the boundedness of $\left\{\bar{x}_{1}(k)\right\},\left\{\bar{x}_{2}(k)\right\},\left\{x_{1}(k)\right\},\left\{x_{2}(k)\right\}, g^{\prime}\left(\xi_{6}^{\prime}(k)\right)$ is bounded, where $g(x)=x^{p} /\left(1+m x^{p}\right)$ and $g^{\prime}$ means the derivation of the function $g(x)$. Let $\left|g^{\prime}\left(\xi_{6}^{\prime}(k)\right)\right|<W_{3}$.

Also, one has

$$
\begin{aligned}
v_{1}(k+1) & =\left(1-\eta_{1}(k)\right) v_{1}(k)+q_{1}(k) \bar{x}_{1}(k)\left(\exp \left\{y_{1}(k)\right\}-1\right) \\
& =\left(1-\eta_{1}(k)\right) v_{1}(k)+q_{1}(k) \bar{x}_{1}(k) \exp \left\{\xi_{4}(k) y_{1}(k)\right\} y_{1}(k), \\
v_{2}(k+1) & =\left(1-\eta_{2}(k)\right) v_{2}(k)+q_{2}(k) \bar{x}_{2}(k)\left\{\left(\exp \left\{y_{2}(k)\right\}-1\right\}\right) \\
& =\left(1-\eta_{2}(k)\right) v_{2}(k)+q_{2}(k) \bar{x}_{2}(k) \exp \left\{\xi_{5}(k) y_{2}(k)\right\} y_{2}(k) .
\end{aligned}
$$

In view of (4.5)-(4.8), we can choose a $\varepsilon>0$ such that

$$
\begin{aligned}
& \lambda_{1}^{\varepsilon}=\max \left\{\left|1-\theta b_{1}^{L}\left(x_{1 *}-\varepsilon\right)^{\theta}\right|,\left|1-\theta b_{1}^{M}\left(x_{1}^{*}+\varepsilon\right)^{\theta}-\alpha_{1}^{M} W_{1}\left(x_{1}^{*}+\varepsilon\right)\right|\right\}+\alpha_{1}^{M} W_{2}\left(x_{2}^{*}+\varepsilon\right)+e_{1}^{M}<1, \\
& \lambda_{2}^{\varepsilon}=\max \left\{\left|1-b_{2}^{L}\left(x_{2 *}-\varepsilon\right)\right|,\left|1-b_{2}^{M}\left(x_{2}^{*}+\varepsilon\right)\right|\right\}+\alpha_{2}^{M} W_{3}\left(x_{1}^{*}+\varepsilon\right)+e_{2}^{M}<1, \\
& \lambda_{3}^{\varepsilon}=1-\eta_{1}^{L}+q_{1}^{M}\left(x_{1}^{*}+\varepsilon\right)<1 \\
& \lambda_{4}^{\varepsilon}=1-\eta_{2}^{L}+q_{2}^{M}\left(x_{2}^{*}+\varepsilon\right)<1 .
\end{aligned}
$$

Also, from Propositions 3.1 and 3.2, there exist $K_{3}>K_{2}$ such that

$$
x_{i *}-\varepsilon \leq x_{i}(k), \quad x_{i}^{*}(k) \leq x_{i}^{*}+\varepsilon \quad \forall k \geq K_{3}, i=1,2 .
$$


Then from (4.11), for $k>K_{3}$, one has

$$
\begin{aligned}
\left|y_{1}(k+1)\right| \leq & \max \left\{\left|1-\theta b_{1}^{L}\left(x_{1 *}-\varepsilon\right)^{\theta}\right|,\left|1-\theta b_{1}^{M}\left(x_{1}^{*}+\varepsilon\right)^{\theta}-\alpha_{1}^{M} W_{1}\left(x_{1}^{*}+\varepsilon\right)\right|\right\} \\
& \cdot\left|y_{1}(k)\right|+\alpha_{1}^{M} W_{2}\left(x_{2}^{*}+\varepsilon\right)\left|y_{2}(k)\right|+e_{1}^{M}\left|v_{1}(k)\right| .
\end{aligned}
$$

So from (4.13), for $k>K_{3}$, one has

$$
\begin{aligned}
\left|y_{2}(k+1)\right| \leq & \max \left\{\left|1-b_{2}^{L}\left(x_{2 *}-\varepsilon\right)\right|,\left|1-b_{2}^{M}\left(x_{2}^{*}+\varepsilon\right)\right|\right\}\left|y_{2}(k)\right| \\
& +\alpha_{2}^{M} W_{3}\left(x_{1}^{*}+\varepsilon\right)\left|y_{1}(k)\right|+e_{2}^{M}\left|v_{2}(k)\right| .
\end{aligned}
$$

Also, for $k>K_{3}$, one has

$$
\begin{aligned}
& \left|v_{1}(k+1)\right| \leq\left(1-\eta_{1}^{L}\right)\left|v_{1}(k)\right|+q_{1}^{M}\left(x_{1}^{*}+\varepsilon\right)\left|y_{1}(k)\right|, \\
& \left|v_{2}(k+1)\right| \leq\left(1-\eta_{2}^{L}\right)\left|v_{2}(k)\right|+q_{2}^{M}\left(x_{2}^{*}+\varepsilon\right)\left|y_{2}(k)\right| .
\end{aligned}
$$

Let $\lambda=\max \left\{\lambda_{1}^{\varepsilon}, \lambda_{2}^{\varepsilon}, \lambda_{3}^{\varepsilon}, \lambda_{4}^{\varepsilon}\right\}$, then $0<\lambda<1$. In view of (4.18)-(4.21), one has

$$
\max \left\{\left|y_{1}(k+1)\right|,\left|y_{2}(k+1)\right|,\left|v_{1}(k+1)\right|,\left|v_{2}(k+1)\right|\right\} \leq \lambda \max \left\{\left|y_{1}(k)\right|,\left|y_{2}(k)\right|,\left|v_{1}(k)\right|,\left|v_{2}(k)\right|\right\}
$$

for $k>K_{3}$. This implies

$$
\max \left\{\left|y_{1}(k)\right|,\left|y_{2}(k)\right|,\left|v_{1}(k)\right|,\left|v_{2}(k)\right|\right\} \leq \lambda^{k-K_{3}} \max \left\{\left|y_{1}\left(K_{3}\right)\right|,\left|y_{2}\left(K_{3}\right)\right|,\left|v_{1}\left(K_{3}\right)\right|,\left|v_{2}\left(K_{3}\right)\right|\right\} .
$$

Therefore

$$
\lim _{k \rightarrow \infty} y_{i}(k)=0, \quad \lim _{k \rightarrow \infty} v_{i}(k)=0, \quad i=1,2
$$

This completes the proof.

\section{Examples}

The following two examples show the feasibility of our main results.

Example 5.1. Consider system (1.3) with

$$
\begin{gathered}
r_{1}(k)=0.14+0.01 \cos (k), \quad b_{1}(k)=0.1, \quad \alpha_{1}(k)=0.001, \quad e_{1}(k)=0.03+0.01 \sin (k), \\
r_{2}(k)=0.18+0.02 \cos (\sqrt{2} k), \quad b_{2}(k)=1.8+0.1 \sin (k), \quad \alpha_{2}(k)=1.4, \\
e_{2}(k)=0.008+0.002 \sin (k), \quad \eta_{1}(k)=0.7, \quad q_{1}(k)=0.2+0.1 \sin (k), \quad \eta_{2}(k)=0.8, \quad \quad \quad \quad \tau_{1}=\tau_{2}=\tau_{3}=\tau_{4}=1, \quad p=1.3, \quad \theta=1.2, \quad m=0.8,
\end{gathered}
$$




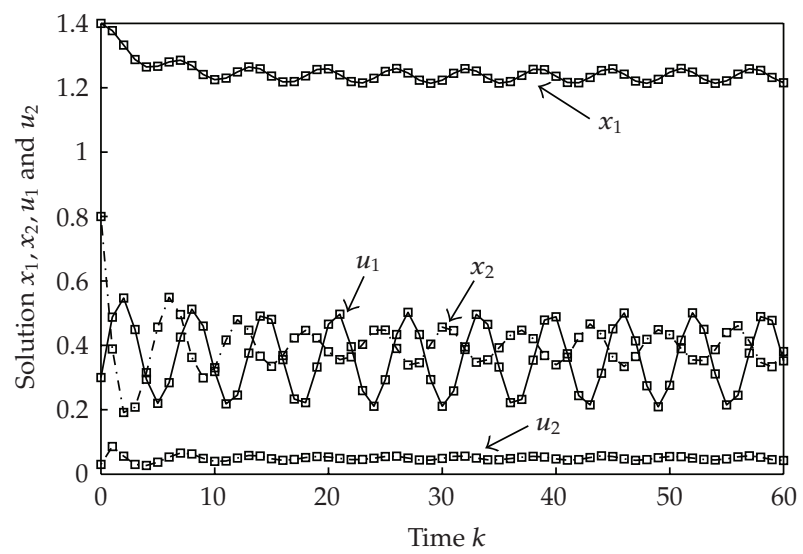

Figure 1: Dynamics behaviors of system (1.3) with initial condition $\left(x_{1}(p), x_{2}(p), u_{1}(p), u_{2}(p)\right)=(1.4,0.8$, $0.3,0.03)(P=-1,0)$.

for all $k \in N$. One can easily see that

$$
x_{1}^{*} \approx 3.7945, \quad x_{1 *} \approx 0.2882, \quad x_{2}^{*} \approx 5.2037, \quad u_{1}^{*} \approx 1.6262, \quad u_{2}^{*} \approx 0.6505,
$$

which means that

$$
\begin{aligned}
& r_{1}^{L}-\alpha_{1}^{M}\left(x_{1}^{*}\right)^{p-1} x_{2}^{*}-e_{1}^{M} u_{1}^{*} \approx 0.0872, \\
& -r_{2}^{M}+\frac{\alpha_{2}^{L} x_{1 *}^{p}}{1+m x_{1 *}^{p}}-e_{2}^{M} u_{2}^{*} \approx 0.0333 .
\end{aligned}
$$

Also, one has

$$
\left(\frac{\alpha_{2}(k)}{m}-r_{2}(k)\right)^{L} \approx 1.55
$$

Inequalities (5.3)-(5.5) show that $\left(H_{1}\right)-\left(H_{3}\right)$ are fulfilled. From Theorem 3.3, system (1.3) is permanent. Figure 1 is the numeric simulation of the solution of system (1.3) with initial condition $\left(x_{1}(p), x_{2}(p), u_{1}(p), u_{2}(p)\right)=(1.4,0.8,0.3,0.03)(P=-1,0)$.

Example 5.2. Consider system (4.1) with

$$
\begin{aligned}
& r_{1}(k)=0.13+0.02 \cos (k), \quad b_{1}(k)=0.1, \quad \alpha_{1}(k)=0.01, \quad e_{1}(k)=0.03+0.01 \sin (k), \\
& r_{2}(k)=0.16+0.02 \cos (k), \quad b_{2}(k)=0.7, \quad \alpha_{2}(k)=0.6, \quad e_{2}(k)=0.015+0.005 \sin (k), \\
& \eta_{1}(k)=0.7, \quad q_{1}(k)=0.2+0.1 \sin (k), \quad \eta_{2}(k)=0.8, \quad q_{2}(k)=0.2+0.1 \sin (k),
\end{aligned}
$$




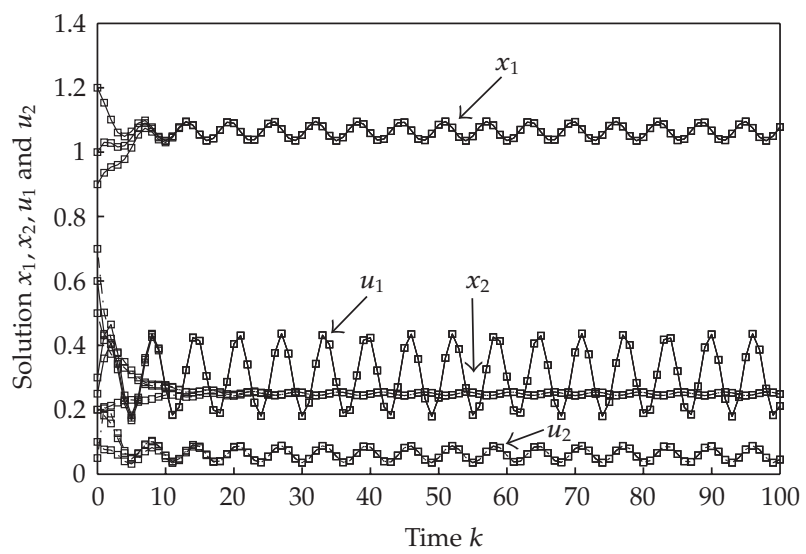

Figure 2: Dynamics behaviors of system (4.1) with initial values $\left(x_{1}(0), x_{2}(0), u_{1}(0), u_{2}(0)\right)=(0.9,0.7,0.6$, $0.05),(1.2,0.5,0.3,0.2)$, and $(1,0.2,0.25,0.1)$.

for all $k \in N$. One can easily see that

$$
x_{1}^{*} \approx 1.2808, \quad x_{1 *} \approx 0.8195, \quad x_{2}^{*} \approx 0.9672, \quad x_{2 *} \approx 0.1157, \quad u_{1}^{*} \approx 0.5489, \quad u_{2}^{*} \approx 0.3627,
$$

which means that

$$
\begin{aligned}
& r_{1}^{L}-\alpha_{1}^{M}\left(x_{1}^{*}\right)^{p-1} x_{2}^{*}-e_{1}^{M} u_{1}^{*} \approx 0.0801, \\
& -r_{2}^{M}+\frac{\alpha_{2}^{L} x_{1 *}^{p}}{1+m x_{1 *}^{p}}-e_{2}^{M} u_{2}^{*} \approx 0.1387
\end{aligned}
$$

Also, one has

$$
\left(\frac{\alpha_{2}(k)}{m}-r_{2}(k)\right)^{L} \approx 0.5700
$$

Inequalities (5.8)-(5.10) show that $\left(H_{1}\right)-\left(H_{3}\right)$ are fulfilled. We can obtain that

$$
W_{1} \approx 0.7258, \quad W_{2} \approx 0.6630, \quad W_{3} \approx 0.075,
$$

which means that

$$
\lambda_{1} \approx 0.8861, \quad \lambda_{2} \approx 0.9966, \quad \lambda_{3} \approx 0.6842, \quad \lambda_{4} \approx 0.4902 .
$$

So (4.5)-(4.8) are fulfilled. From Theorem 4.2, system (4.1) is globally attractive. Figure 2 is the numeric simulation of the solution of system (4.1) with initial condition $\left(x_{1}(0)\right.$, $\left.x_{2}(0), u_{1}(0), u_{2}(0)\right)=(0.9,0.7,0.6,0.05),(1.2,0.5,0.3,0.2)$, and $(1,0.2,0.25,0.1)$. 


\section{Acknowledgment}

This work was supported by the Foundation of Fujian Education Bureau (JB05042).

\section{References}

[1] C. S. Holling, "The components of predation as revealed by a study of small mammal predation of the European pine sawfly," Canadian Entomologist, vol. 91, pp. 293-320, 1959.

[2] C. S. Holling, "Some characteristics of simple types of predation and parasitism," Canadian Entomologist, vol. 91, pp. 385-398, 1959.

[3] M. Fan and K. Wang, "Global existence of positive periodic solutions of a predator-prey system with Holling type II functional response," Acta Mathematica Scientia, vol. 21, no. 4, pp. 492-497, 2001.

[4] L. Yang, "Periodic solutions of a delayed discrete predator-prey system with general functional response," Journal of Systems Science and Mathematical Sciences, vol. 27, no. 4, pp. 488-498, 2007 (Chinese).

[5] M. Fan and K. Wang, "Periodic solutions of a discrete time nonautonomous ratio-dependent predatorprey system," Mathematical and Computer Modelling, vol. 35, no. 9-10, pp. 951-961, 2002.

[6] F. Chen, L. Wu, and Z. Li, "Permanence and global attractivity of the discrete Gilpin-Ayala type population model," Computers Mathematics with Applications, vol. 53, no. 8, pp. 1214-1227, 2007.

[7] Y. Li, "Positive periodic solutions of discrete Lotka-Volterra competition systems with state dependent and distributed delays," Applied Mathematics and Computation, vol. 190, no. 1, pp. 526-531, 2007.

[8] Y. Chen and Z. Zhou, "Stable periodic solution of a discrete periodic Lotka-Volterra competition system," Journal of Mathematical Analysis and Applications, vol. 277, no. 1, pp. 358-366, 2003.

[9] Z. Zhou and X. Zou, "Stable periodic solutions in a discrete periodic logistic equation," Applied Mathematics Letters, vol. 16, no. 2, pp. 165-171, 2003.

[10] Y.-H. Fan and W.-T. Li, "Permanence for a delayed discrete ratio-dependent predator-prey system with Holling type functional response," Journal of Mathematical Analysis and Applications, vol. 299, no. 2, pp. 357-374, 2004.

[11] H.-F. Huo and W.-T. Li, "Existence and global stability of periodic solutions of a discrete ratiodependent food chain model with delay," Applied Mathematics and Computation, vol. 162, no. 3, pp. 1333-1349, 2005.

[12] Y. Li and L. Zhu, "Existence of positive periodic solutions for difference equations with feedback control," Applied Mathematics Letters, vol. 18, no. 1, pp. 61-67, 2005.

[13] H.-F. Huo and W.-T. Li, "Positive periodic solutions of a class of delay differential system with feedback control," Applied Mathematics and Computation, vol. 148, no. 1, pp. 35-46, 2004.

[14] Y. Li, "Positive periodic solutions for a periodic neutral differential equation with feedback control," Nonlinear Analysis: Real World Applications, vol. 6, no. 1, pp. 145-154, 2005.

[15] F. Chen, "Positive periodic solutions of neutral Lotka-Volterra system with feedback control," Applied Mathematics and Computation, vol. 162, no. 3, pp. 1279-1302, 2005.

[16] F. Chen, "The permanence and global attractivity of Lotka-Volterra competition system with feedback controls," Nonlinear Analysis: Real World Applications, vol. 7, no. 1, pp. 133-143, 2006.

[17] L. Wang and M. Q. Wang, Ordinary Difference Equation, Xinjiang University Press, Xinjiang, China, 1991. 


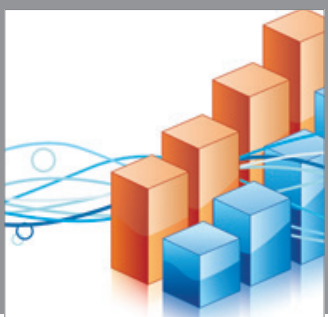

Advances in

Operations Research

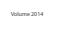

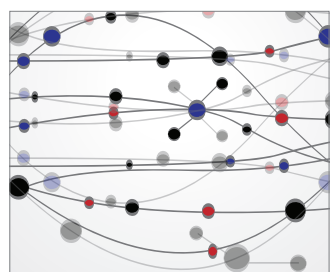

\section{The Scientific} World Journal
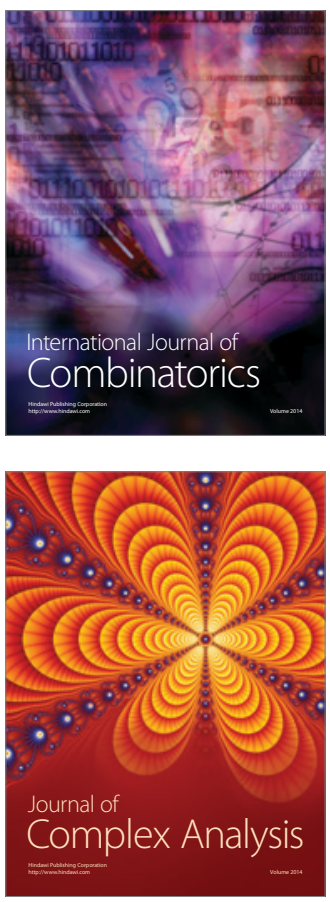

International Journal of

Mathematics and

Mathematical

Sciences
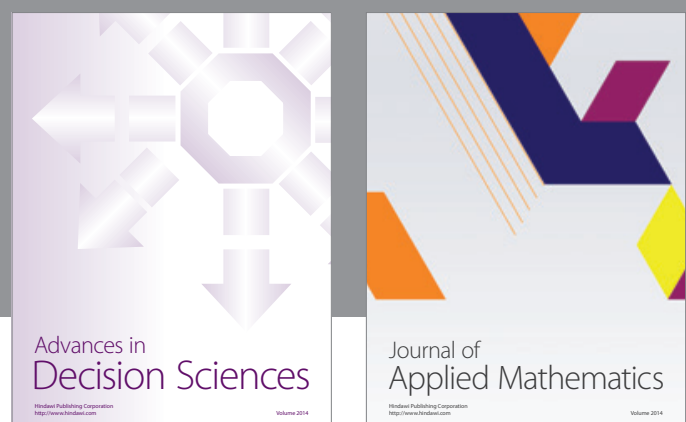

Journal of

Applied Mathematics
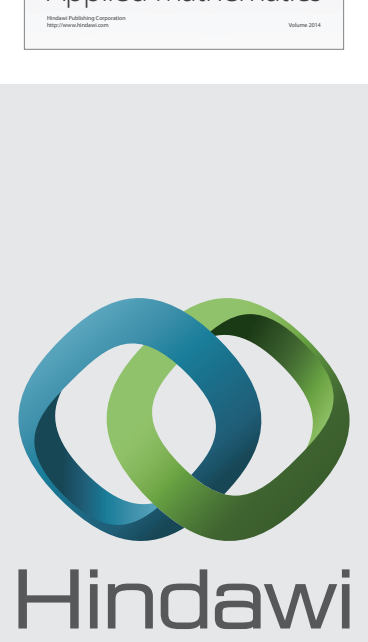

Submit your manuscripts at http://www.hindawi.com
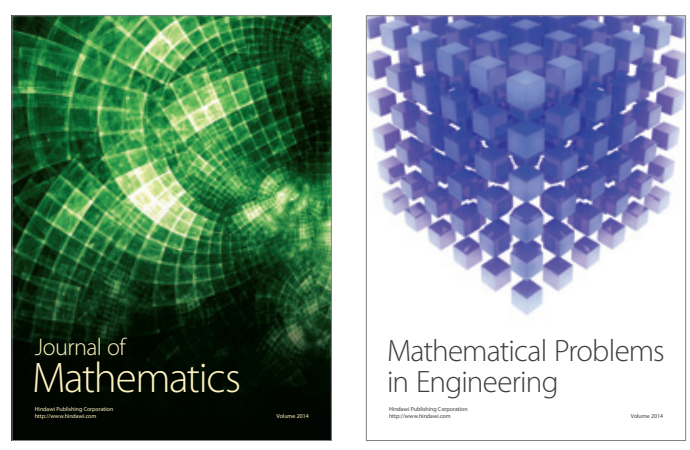

Mathematical Problems in Engineering
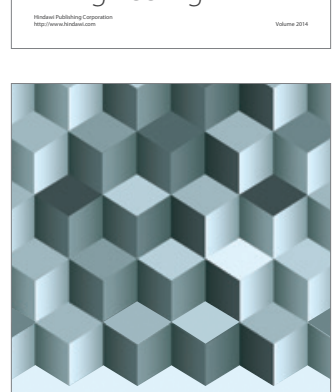

Journal of

Function Spaces
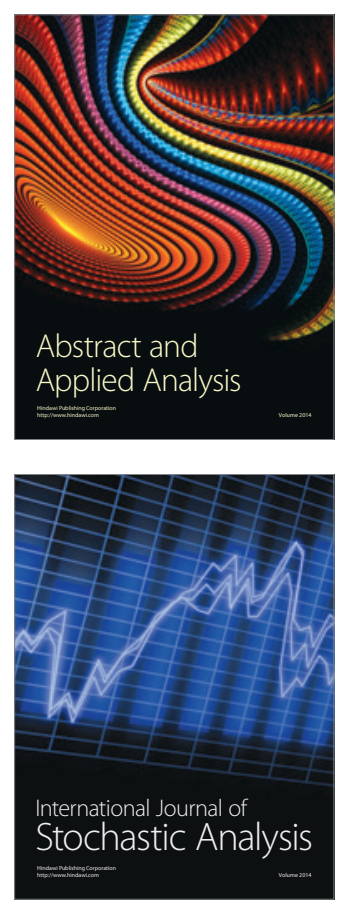

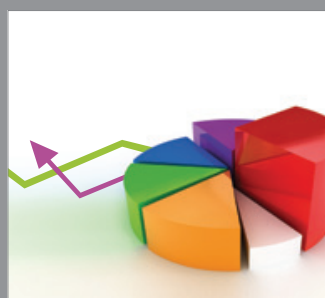

ournal of

Probability and Statistics

Promensencen
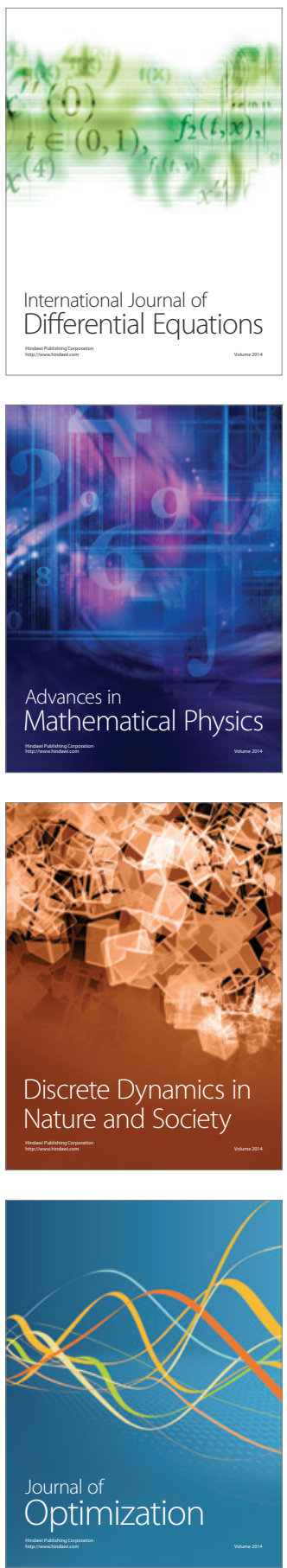